\title{
The Importance of Physics for the Siemens AG
}

\section{W. Heywang, Munich \\ (Head of Siemens Central Research Labs.)}

Physics in the EPS industrial Associate Members, of which Siemens AG is one, will be regularly featured over the coming months.

The first of October in the year 1847 marked the founding of the Telegraphen-Bauanstalt Siemens \& Halske by Werner Siemens, Johann Georg Halske and Johann Georg Siemens as one of the earliest electrical engineering enterprises.

As an inventor of numerous electrical apparatuses, Werner Siemens gave the company of which he was a co-founder a specifically engineering character which for example, is reflected to this day in the fact that despite continuous expansion it still remains dedicated to its original field of pure electrical engineering. Resulting from the merger in 1966 of Siemens \& Halske and its two affiliated companies, Siemens-Schuckert and Siemens-Reiniger, the Siemens AG now covers the broadest electrical engineering spectrum, extending all the way from components through communications systems, data systems and power engineering to medical electronics. In recent years this spectrum has been further extended and rounded off, especially by the complete takeover of the Kraftwerkunion in the sector of large power stations and of Osram in the electrical illumination sector.

With a work force of 319,000 in all parts of the globe and annual sales to the tune of $25 \mathrm{G} \mathrm{DM}$, the Siemens $A G$ is one of the world's largest electrical engineering organizations. As such, it naturally plays an active part in the further development of electrical engineering, its efforts in this direction being evidenced by its capital investment of $2.1 \mathrm{G} \mathrm{DM}$ in research and development, representing more than $8 \%$ of its returns from sales. A great many of its R\&D projects are of course related to product applications and carried out at our product-oriented laboratories, but $11 \%$ of the R\&D work is advisediy allocated to our central laboratories, which are responsible for futureoriented basic research. The overall engineering work at Siemens is performed by 21,000 engineers and natural scientists, about a third of whom have academic degrees; 1200 of these, some $18 \%$, are physicists. They are by no means concerned exclusively with basic research as we see below.

Beginning with basic research per se, it is necessary to review past achievements because their technological relevance can only be properly evaluated after the passage of some years. A typical example is presented by semiconductor technology, where Siemens were responsible for major contributions to the introduction of two groups of semiconductor materials, viz. silicon and $A^{(3)} B^{(5)}$ com pounds, the latter, in particular, showing the length of time which may elapse between a physical discovery and its ultimate technical application to a product. The principal semiconductor properties of this entire group of semiconductor materials - controllable energy band gap, extremely high carrier mobility, light emission induced by carrier recombination were discovered as early as the 1950s by a research team headed by $\mathrm{H}$. Welker at our Erlangen laboratories. The results opened the way in various parts of the world to a number of other related physical discoveries concerning the structure of the energy band in semiconductors. After their initial application, in Hall generators and magnetoresistors, it was not until the 1970s that these compounds achieved a real breakthrough in the field of optoelectronics (LEDs, laser diodes) and GaAs devices for microwave frequencies. This belated development - at Siemens as elsewhere throughout the world - was due to the far more complicated technology of such compounds in comparison with silicon, which was produced in ultrapure quality already in the 1950s.

The properties of this ultrapure silicon are so outstanding, that it is used to the exclusion of all other semiconductor materials whenever possible. This applies to the already noted application areas of $A^{(3)} B^{(5)}$ semiconductor compounds. But why this should be so may not be generally appreciated. Silicon possesses an adequate energy band gap, satisfactory carrier mobility and satisfactory carrier lifetime; in addition to there being controllable purification and crystal growing techniques suitable for mass production, it has high chemical stability and it develops a stable oxide skin.

It is above all this oxide skin which, utilized in a large variety of ways, has opened the way to modern verylarge-scale integration (VLSI) devices.

Distribution of physicists relative to the various branches of activity (in round numbers).

Branch

Research

Development

Production

Assembly, Maintenance

Project planning

Distribution

Miscellaneous
No. of Physicists 160

700

50

30

90

120

\section{Swiss Federal Institute of Technology}

Post-doctoral
Research Position

in

Theoretical

Surface Physics
A two-year position with possible renewal is available for a solid-state theoretician
at the Laboratory of Applied Physics, Swiss Institute of Technology, Lausanne. Applicants should be interested in semiconductor bandstructure calculations and their extension to surfaces and heterojunctions.

Applications enclosing curriculum vitæ, list of publications and the names of two referees should be sent to:

Service du personnel de l'Ecole Polytechnique Fédérale de Lausanne, avenue de Cour 33, 1007 Lausanne / Suisse. 


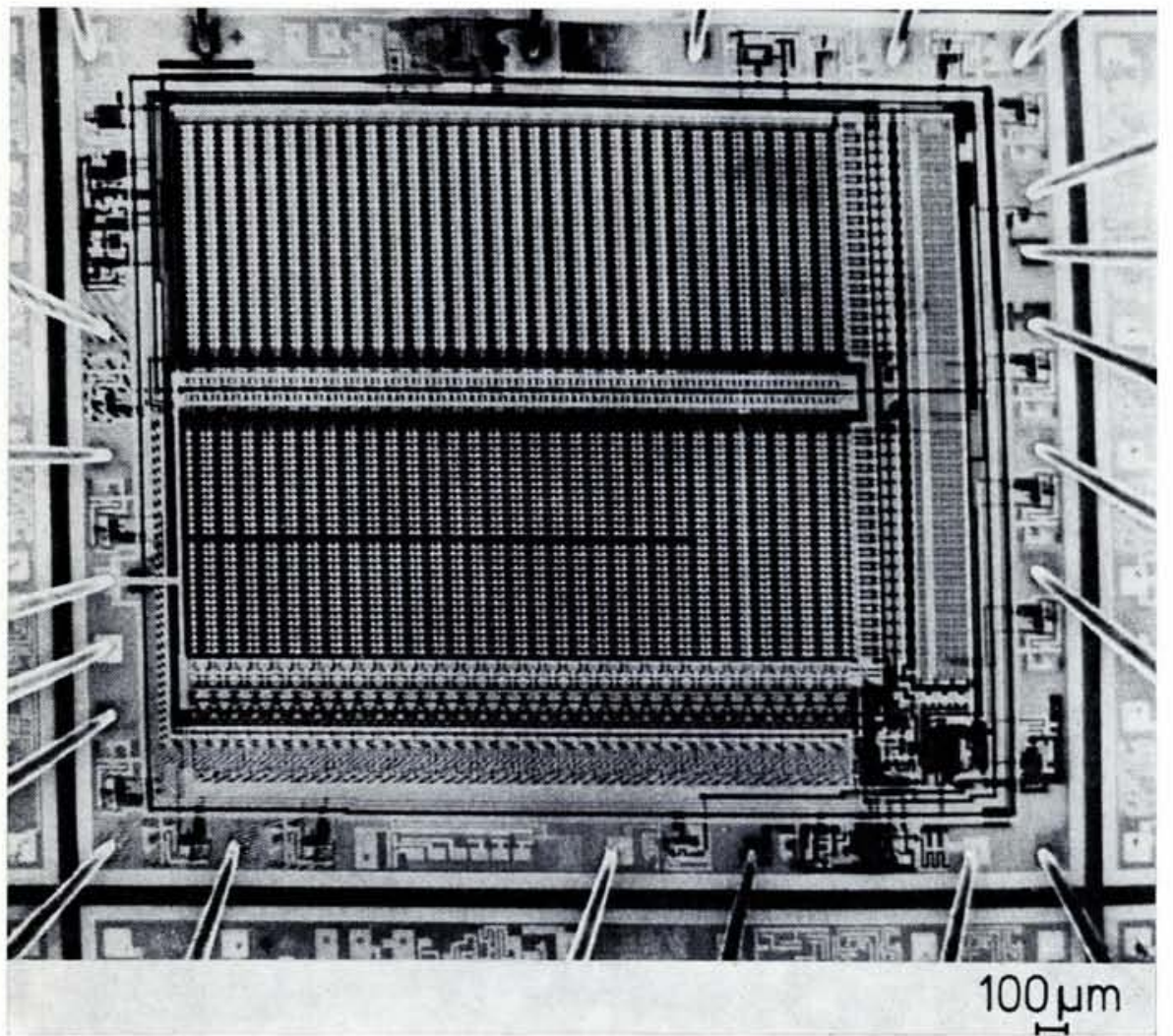

The reason why I am stressing this point is because it goes to show that the method of producing ultrapure silicon represented only one step in the revolution in electronics initiated by this semiconductor material. In this connection, we cooperated with many laboratories in all parts of the world.

Ultrapure silicon was first applied to power electronics based on rectifiers and thyristors, but has since been extended to the integrated circuits used in all branches of electronics which, with their present packing densities of over $10^{5}$ devices per chip, are still by no means anywhere near the end of their capabilities. The extent of our pioneer contributions in the VLSI area is shown by the following example drawn from our analytical work.

The steadily increasing packing density per chip is accompanied by growing difficulties relating to function testing in both development work and in fabrication. Structural widths of the order of $3 \mu \mathrm{m}$ have already been realized and work is in progress with the objective of bringing this figure down to the $1 \mu \mathrm{m}$ mark. Just as these extremely fine structures can only be realized by using the electron beam as a tool, it is likewise practical to use an electron beam as a probe for function testing. As the slow secondary electrons excited by the electron beam incident upon the chip are retarded to a greater or lesser extent as a function of the surface voltage, the secondary electron emission signal can be used for producing an image directly representing the voltage contrast of the specimen. Comparison with an ideal image instantly discloses any defect such as the failure of an array of memory cells due to a discontinuity in a supply line caused by a dust particle. That our laboratories have further succeeded in performing quantitative measurements of these effects under dynamic conditions is of great significance.

The already noted VLSI development brings us immediately one step further by introducing entirely new possibilities in electronics. New areas of application come within reach, while existing areas are revolutionized. Wherever electromechanical components are still used, integrated circuits now offer simpler, more economic and more effective solutions. This applies to the entire spectrum of communications, industrial processing and data systems - three principal fields of Siemens activity. Here, where all is conceived in terms of complete systems, the function of the physicist might be assumed to come to an end. Such is, however, by no means the case. A system has to communicate with its ambiance, a function for which special pickup and output devices need to be developed. One example which comes readily to mind is the microphone and receiver insets of the telephone. Acoustic waves nave to be transduced into electric, and
Fig. 1 - Stroboscopic voltage contrast pattern of a memory circuit. One horizontal word line in the lower cell field exhibits anomalous contrast indicating an aluminium interruption.

electric into acoustic. For this application we developed novel piezoelectric transducers which operate with the low random noise and minimal distortion demanded by modern systems, and require moreover, lower input voltage. Only through the closest cooperation between physicists and systems engineers was it possible to develop and exploit these advantages.

Let us remain for the time being with the example of the piezoelectric transducer because it throws particular light on the professional opportunities for a physicist in an organization which pursues as broad a spectrum of activities as our own.

The piezoelectric transducers developed from ferroelectric materials for communications systems have so many unique properties that they can be employed for many other applications, such as special filters for longhaul communications systems and television, in delay lines for signal processing, as ultrasound transducers for instrumentation, and in medical electronics relating to inhalation, aids for the blind and for ultrasound diagnostics. The physicists who developed these materials were also responsible for discovering and developing new areas of application. Ultrasound diagnostics lead us straight back to thinking in terms of systems, for diagnosis requires the development of not only transducer systems but also appropriate evaluation techniques as well.

Evaluation techniques nowadays imply for the most part adequate software. This heading brings us to a class of problem which is beginning to dominate the entire spectrum of electrical engineering developments, especially data technology and automatic feedback control. Here again it might at first be assumed that the development of such software is exclusively the responsibility of the mathematician and the computer expert. But once again the same applies as in systems engineering. It is no longer possible to make a hard and fast division between software and hardware. The worldwide problems of software can only be solved by mastering all modern varieties of the art of effectively matching the software to the hardware that is made available by large-scale integration and microprocessors.

Once more the physicist has an important function to perform because 
from his earliest studies he has developed a faculty for rigorous analysis and for combining different effects. He knows from experience that a problem can only be solved after it has been properly analyzed on the basis of its boundary conditions. From his early training he will also have acquired a broad overall perspective which enables him to spot a large variety of areas of application or to employ a fundamental approach in tackling problems. And more than enough problems are certainly bound to confront us in the coming years.

In this connection I need only draw attention to the efforts that will be needed to solve the energy problem. It is not only necessary to develop new methods of generating and storing energy, ranging from nuclear to solar energy, but to develop new methods and materials which will allow the more efficient utilization of energy at both low and superhigh temperatures, and all must be integrated into an overall system of power economy that is both technically and economically compatible with the existing economic system. At the same time, the current problems of environmental protection and safety demand an overall perspective based on technological and scientific knowledge which reaches far beyond the specialized knowledge of individual experts.

I have here of course concentrated on the problems of the physicists, but these can be solved only in part by physicists, their solution requiring additionally the cooperation of experts with other types of training and of scientists who work not only in the laboratory but also in production, distribution, etc. It is of some interest to note, as shown in the Table, the large percentage of physicists that ultimately find their fulfilment in branches of activity outside R\&D.

Due to the current interaction of different branches of engineering, a knowledge of physics is important not only in research \& development but is equally important in solving problems in production, distribution and patent law.

It goes without saying that the broad spectrum of problems encountered in a steadily advancing technology can only be solved if physicists are prepared to acquire capabilities and knowledge beyond what they have learned at college. Siemens accordingly offers a large variety of refresher courses for the benefit of physicists with their already extensive basic training. Thus it is not surprising that physicists are to be found in responsible positions where new

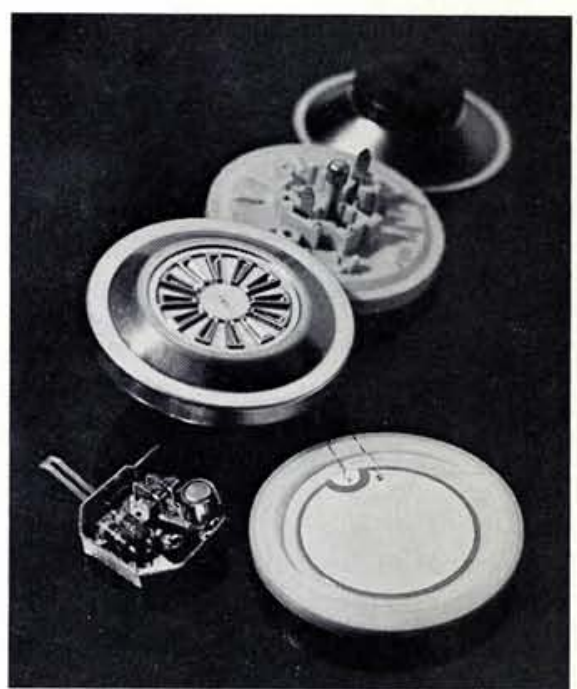

Fig. 2 - Siemens piezo microphone.

types of project need to be managed and a broad knowledge of various different scientific and technological disciplines is essential.

Finally it will be noted from the foregoing that electrical engineering is currently more closely allied with physics than ever before, so that optimal interaction between the two is essential for the whole of the future and consequently also for a vast electrical engineering organization such as the Siemens $A G$ with its worldwide responsibilities.

\section{The International Laboratory of High Magnetic Fields and Low Temperatures in Wroclaw

The proposal to create the International Laboratory of High Magnetic Fields and Low Temperatures (I.L.) emerged for the first time at a meeting of COMECON experts on low temperature physics in 1964. Subsequently, Wroclaw in Poland was proposed as the site, as the necessary DC power sources already existed there, in the form of a substation of the LowSilesian Power Plant. This station which was presented to the Polish Academy of Sciences comprises several generators with a total capacity of $5.5 \mathrm{MW}$. On 11 May 1968, the representatives of the Bulgarian Academy of Sciences, the Academy of Sciences in the GDR, the Polish Academy of Sciences and the Academy of Sciences of USSR signed the agreement that set up the Laboratory and approved the Statutes and Regulations.

I.L. is a legally independent organi- zation supported by the four Academies and is supervised by a Council on which each is represented by two places. The Chairman of the Council is elected for a term of two years and, on a proposal submitted by the Polish Academy of Sciences, the Director of the I.L. for a term of three years. The Deputy Director is again elected but for a term of two years. The Director is directly responsible for the activity of the I.L. to Council, which each year establishes the budget and the scientific research programme. It also accepts the annual scientific and financial reports of the directorate, the last being examined by a Control Committee on which each Academy has one representative. The chairman is appointed in rotation.

The Council meets twice a year and its resolutions are passed by simple majority, each Academy casting one vote. Since its foundation, the
Chairman of Council has been Professor N.Eu. Aleksejevskii, and the position of Director held by the author. As Deputy Director, Prof. F. Lange (GDR), Dr. K. Berthel (GDR), Dr. Eu Leyarovskii (BDR) and recently $\mathrm{Dr}$. Cz. Bazan (Poland) have acted in turn.

\section{Major Facilities}

It was decided that the first aim should be to make available a steady magnetic field of $20 \mathrm{~T}$ which was achieved progressively with the construction of three Bitter magnets designed by K. Trojnar. The characteristics of these magnets (I-III) are given in the following table:

Field stability is about $10^{-3}$. Using dysprosium or permendur concentrators, it is possible also to increase the field strength by at least $2 \mathrm{~T}$. In parallel, the cooling system was extended and now includes two circuits. The primary closed system contains deionized water at $17 \mathrm{~atm}$ pressure, 